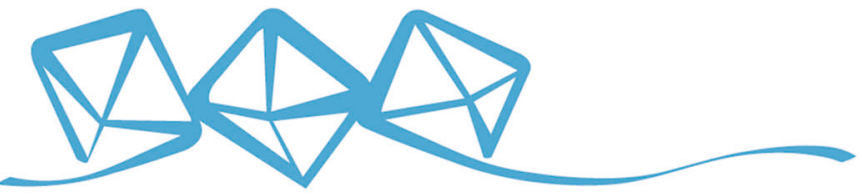 COMMUNICATIONS MATERIALS
}

ARTICLE

https://doi.org/10.1038/s43246-020-00076-0 OPEN

\section{Large spin-Hall effect in non-equilibrium binary copper alloys beyond the solubility limit}

Hiroto Masuda (1) 1,6, Rajkumar Modak (1) 2,6, Takeshi Seki1,2,3凶 , Ken-ichi Uchida (10) 1,2,3凶 , Yong-Chang Lau (1) 1,3, Yuya Sakuraba (1) ${ }^{2,4}$, Ryo Iguchi (i) ${ }^{2}$ \& Koki Takanashi, ${ }^{1,3,5}$

Non-magnetic materials exhibiting large spin-Hall effect (SHE) are eagerly desired for highperformance spintronic devices. Here, we report that non-equilibrium Cu-Ir binary alloys with compositions beyond the solubility limit are candidates as spin-Hall materials, even though $\mathrm{Cu}$ and Ir do not exhibit remarkable SHE themselves. Thanks to non-equilibrium thin film fabrication, the $\mathrm{Cu}-\mathrm{Ir}$ binary alloys are obtained over a wide composition range even though they are thermodynamically unstable in bulk form. We investigate the SHE of Cu-lr by exploiting a combinatorial technique based on spin Peltier imaging, and find that the optimum Ir concentration for enhancing SHE is around 25 at.\%. We achieve a large spin-Hall angle of $6.29 \pm 0.19 \%$ for $\mathrm{Cu}_{76} \mathrm{Ir}_{24}$. In contrast to Cu-Ir, non-equilibrium Cu-Bi binary alloys do not show remarkable SHE. Our discovery opens a new direction for the exploration of spin-Hall materials.

\footnotetext{
${ }^{1}$ Institute for Materials Research, Tohoku University, Sendai 980-8577, Japan. ${ }^{2}$ Research Center for Magnetic and Spintronic Materials, National Institute for Materials Science, Tsukuba 305-0047, Japan. ${ }^{3}$ Center for Spintronics Research Network, Tohoku University, Sendai 980-8577, Japan. ${ }^{4}$ PRESTO, Japan Science and Technology Agency, Saitama 332-0012, Japan. ${ }^{5}$ Center for Science and Innovation in Spintronics, Core Research Cluster, Tohoku University, Sendai 980-8577, Japan. ${ }^{6}$ These authors contributed equally: Hiroto Masuda, Rajkumar Modak. ${ }^{凶}$ email: go-sai@imr.tohoku.ac.jp; UCHIDA.Kenichi@nims.go.jp
} 
G eneration and detection of spin current $\left(\mathbf{J}_{\mathrm{s}}\right)$, which is the flow of spin angular momentum, are the keys for spintronics. In order to improve the device performance, highly efficient conversion between charge current $\left(\mathbf{J}_{\mathrm{c}}\right)$ and $\mathbf{J}_{\mathrm{s}}$ is indispensable. A way for the conversion from $\mathbf{J}_{\mathrm{c}}$ to $\mathbf{J}_{\mathrm{s}}$ is to exploit the spin-Hall effect (SHE) ${ }^{1-5}$, which is expressed as

$$
\mathbf{j}_{\mathrm{s}}=\left(\frac{\hbar}{2 e}\right) \alpha_{\mathrm{SH}}\left[\mathbf{s} \times \mathbf{j}_{\mathrm{c}}\right]
$$

where $\mathbf{j}_{\mathrm{c}}$ and $\mathbf{j}_{\mathrm{s}}$ are charge current density and spin current density, respectively, $\alpha_{\mathrm{SH}}$ is the spin-Hall $(\mathrm{SH})$ angle, $e(<0)$ is the electric charge of an electron, $\hbar$ is the reduced Planck constant, and $\mathbf{s}$ is the quantization axis of electron spin. When $\mathbf{J}_{\mathrm{c}}$ flows in a nonmagnet with large spin-orbit interaction, up-spin and downspin electrons are scattered in opposite directions. This results in a $\mathbf{J}_{\mathrm{s}}$ flow without a net charge current flow in the transverse direction to $\mathbf{J}_{c}$. Equation (1) means that $\alpha_{\mathrm{SH}}$ corresponds to the conversion efficiency, and a nonmagnet showing large $\alpha_{\mathrm{SH}}$ is a building block of contemporary spintronics.

Many recent studies have been devoted to the materials development to get larger $\alpha_{\mathrm{SH}}$, and found a variety of SH materials, which are not only limited to nonmagnetic metals $s^{5}$ but also include topological insulators ${ }^{6,7}$, and ferromagnets ${ }^{8-13}$. Among them, heavy metals such as $\mathrm{Pt}^{14-16}, \mathrm{Ta}^{17,18}$, and $\mathrm{W}^{19}$ are representatives of SH materials at present because those simple heavy metals have potential to be incorporated into the existing spintronic device architecture. Apart from the usage of elemental nonmagnetic metals, element doping and alloying are also effective ways to develop $\mathrm{SH}$ materials, e.g. $\mathrm{Cu}-\mathrm{Ir}^{20-24}, \mathrm{Cu}-\mathrm{Bi}^{25}$, $\mathrm{Cu}-\mathrm{Pt}^{26-28}, \mathrm{Au}-\mathrm{Pt}^{29,30}$, and $\mathrm{Au}-\mathrm{W}^{31}$. Cu-Ir system is an interesting $\mathrm{SH}$ material because neither $\mathrm{Cu}$ nor Ir exhibits remarkable $\mathrm{SHE}^{32}$. Niimi and co-workers ${ }^{20}$ investigated the SHE of Ir-doped $\mathrm{Cu}$ with the Ir concentration range between $1 \%$ and $12 \%$, which exhibited the large $\alpha_{\mathrm{SH}}$ of $\sim 2.1 \%$. They mentioned that the predominant mechanism of SHE for the Ir-doped $\mathrm{Cu}$ was skew scattering. It was also reported that the $\mathrm{Cu}$ doped with a very small amount of $\mathrm{Bi}(<0.5 \%)$ also shows even larger $\mathrm{SHE}^{25}$. These $\mathrm{Cu}$-based SH materials are also advantageous from the viewpoint of practical applications because of its compatibility to the standard integrated circuit interconnection technology ${ }^{21}$. In spite of the attracting features of Cu-based binary alloys, the comprehensive study on SHE for them is very limited. This limitation for the $\mathrm{Cu}-\mathrm{Ir}$ might be because the solubility limits are narrow at both $\mathrm{Cu}$-rich and Ir-rich sides, which are $<10 \%$, in the $\mathrm{Cu}-\mathrm{Ir}$ binary phase diagram ${ }^{33}$. Although Cramer et al. ${ }^{23}$ recently reported the investigation of SHE for the $\mathrm{Cu}-\mathrm{Ir}$ alloys in a wide composition range, the magnitude and the mechanism of SHE, and the detailed structures have not been understood for the nonequilibrium $\mathrm{Cu}-\mathrm{Ir}$ alloys beyond the solubility limit.

In this paper, we report a comprehensive study on the SHE of $\mathrm{Cu}-\mathrm{Ir}$ binary alloys by combining "high-throughput screening based on thermal imaging for composition-spread films" and "accurate evaluation using harmonic Hall voltage measurement". We utilize the spin Peltier effect (SPE) ${ }^{34,35}$, which is the phenomenon of a heat current generation in linear response to $\mathbf{J}_{s}$ injection in a magnetic insulator/SH material junction, as a probe of the spin-charge current conversion in the $\mathrm{SH}$ material layer since the magnitude and the sign of temperature modulation due to the SPE $\left(\Delta T^{S P E}\right)$ are determined by the spin-charge current conversion. The active infrared emission microscopy called the lock-in thermography (LIT) ${ }^{35-41}$ allows us to visualize $\Delta T^{\mathrm{SPE}}$, and to reveal the spatial distribution of $\Delta T^{\mathrm{SPE}}$ in the compositionspread films. We show the magnitude of SHE is maximized at the non-equilibrium phase that is not thermodynamically stable in the bulk phase diagram. After the high-throughput screening of
SH material, the $\alpha_{\mathrm{SH}}$ value at the optimum composition is evaluated. In addition to the $\mathrm{Cu}-\mathrm{Ir}$, the non-equilibrium $\mathrm{Cu}-\mathrm{Bi}$ binary alloys are also prepared and their SHE is examined for comparison.

\section{Results}

Structure of $\mathrm{Cu}-\mathrm{Ir}$ composition-spread film. The $\mathrm{Cu}-\mathrm{Ir}$ composition-spread films were prepared on a ferrimagnetic yttrium-iron garnet (YIG) substrate with the size of $10 \mathrm{~mm} \times$ $10 \mathrm{~mm}$ by the combinatorial sputtering system (CMS-3200, Comet, Inc.). A schematic illustration of the film sample is displayed in Fig. 1a. The wedge-shaped $\mathrm{Cu}$ and Ir layers were alternately deposited using the linear moving shutter and the rotating substrate holder. After depositing one wedge-shaped layer the substrate was rotated by $180^{\circ}$, and the next wedgeshaped layer was deposited. The thickest part in each layer was designed to be $0.5 \mathrm{~nm}$. In other word, one $\mathrm{Cu} / \mathrm{Ir}$ pair has the thickness of $0.5 \mathrm{~nm}$, where the $\mathrm{Cu}$ and Ir layers were naturally mixed without any heating process. Finally, we obtained the composition-spread films having the Ir concentration $\left(x_{\mathrm{Ir}}\right)$ range from 0 at.\% (pure $\mathrm{Cu}$ ) to 100 at.\% (pure Ir). By repeating the deposition of $0.5 \mathrm{~nm}$-thick $\mathrm{Cu} / \mathrm{Ir}$ pair 20 times, 40 times, and 60 times, the samples with the total thicknesses $(t)$ of 10, 20, and $30 \mathrm{~nm}$, respectively, were obtained. An important point here is that all the layers were deposited at room temperature in order to prevent from the appearance of thermodynamically stable phase. In the blanket film sample used for structural characterization, the gradient of composition was formed in the length of $8.0 \mathrm{~mm}$, which is laterally sandwiched with the pure $\mathrm{Cu}$ and $\mathrm{Ir}$ regions, i.e. $0.6 \mathrm{~mm}$ for the pure $\mathrm{Cu}, 8.0 \mathrm{~mm}$ for the composition-spread region, and $0.6 \mathrm{~mm}$ for the pure Ir. The position in the blanket film $\left(y_{\mathrm{F}}\right)$ was defined as shown in Fig. 1a.

Figure $1 \mathrm{~b}$ shows the $y_{\mathrm{F}}$ dependence of Ir concentration $\left(x_{\mathrm{Ir}}\right)$ for the $\mathrm{Cu}-\mathrm{Ir}$ composition-spread film with $t=30 \mathrm{~nm}$ measured by electron probe x-ray microanalysis (EPMA). It is confirmed that $x_{\mathrm{Ir}}$ is varied with the position. Although in the Cu-rich region $\left(y_{\mathrm{F}} \leq 4 \mathrm{~mm}\right) x_{\mathrm{Ir}}$ almost linearly increases with $y_{\mathrm{F}}$, the position dependence deviates from the tendency in the Ir-rich region $\left(y_{\mathrm{F}} \geq\right.$ $5 \mathrm{~mm}$ ). At present, we have no clear explanation for this rapid increase of $x_{\mathrm{Ir}}$ at the Ir-rich region. The x-ray diffraction (XRD) profiles for $t=30 \mathrm{~nm}$ measured at different $y_{\mathrm{F}}$ are shown in Fig. 1c, where the observed peaks come from the $\mathrm{Cu}-\mathrm{Ir} 111$ reflections. It is noted that the XRD peak angle continuously shifts as $y_{\mathrm{F}}$ is varied, i.e. $x_{\mathrm{Ir}}$ is increased. In addition, no remarkable peak splitting is observed at all the positions. These facts suggest that the lattice constant of single-phase alloys is continuously varied. In other words, the $\mathrm{Cu}-\mathrm{Ir}$ solid solutions are formed at the overall compositions, and the non-equilibrium phase appears at the compositions that are out of the solubility limit of the bulk phase diagram. Figure 1d displays the $y_{\mathrm{F}}$ dependence of the lattice constant $a$. The $y_{\mathrm{F}}$ dependence of $a$ shows the continuous change, and the plot of $a$ versus $x_{\mathrm{Ir}}$ in the inset of Fig. 1d suggests the linear relationship between $a$ and $x_{\text {Ir }}$, meaning that the Vegard's law ${ }^{42}$ is satisfied. Figure le shows the cross-sectional high-resolution transmission electron microscope image for $t=30 \mathrm{~nm}$ together with the element analysis of $\mathrm{Cu}$ and Ir by the energy-dispersive $\mathrm{x}$-ray spectroscopy for the film samples at $y_{\mathrm{F}}=3 \mathrm{~mm}$. From the element mappings, the $\mathrm{Cu}$ and $\mathrm{Ir}$ atoms are uniformly distributed. Therefore, one sees that neither segregation nor phase separation exists remarkably.

Thermal imaging of SPE. In order to evaluate the SHE of $\mathrm{Cu}-\mathrm{Ir}$ composition-spread films, we carried out the SPE measurement using the LIT. As reported previously ${ }^{41}$, this LIT-based SPE measurement enables us to do systematic and high-throughput 


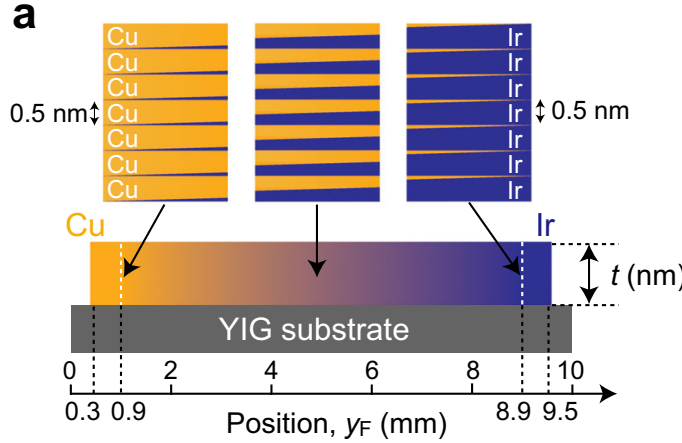

e

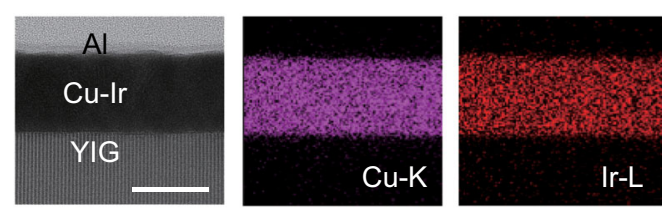

b
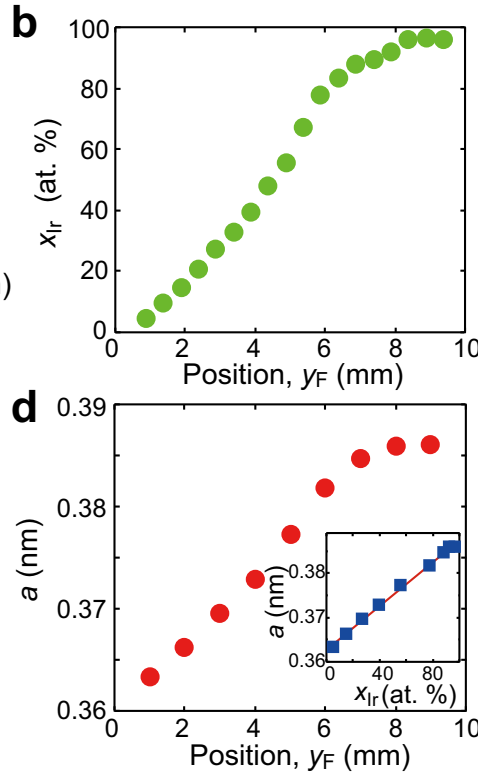

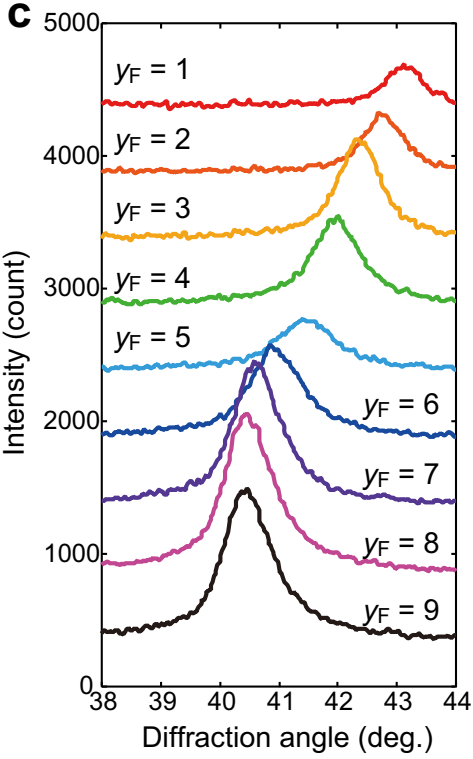

Fig. 1 Structure of Cu-Ir composition-spread film. a Schematic illustration of the blanket film sample used for the structural characterization, where the wedge-shaped $\mathrm{Cu}$ and Ir layers were alternatively deposited using the linear moving shutter on the yttrium-iron-garnet (YIG) substrate. $y_{F}$ is the position in the blanket film. b $y_{F}$ dependence of $x_{\mid r}$ for the Cu-Ir composition-spread film with the thickness ( $t$ ) of 30 nm measured by electron probe $x$-ray microanalysis. c X-ray diffraction profiles for $t=30 \mathrm{~nm}$ measured at different $y_{\mathrm{F}}$. The profiles are vertically shifted for clarity. $\mathbf{d}$ Lattice constant $a$ obtained from the Cu-Ir 111 reflection as a function of $y_{F}$. The inset shows a versus $x_{1 r}$. e (left) Cross-sectional high-resolution transmission electron microscope image, where the length of write scale bar is $25 \mathrm{~nm}$, (center) element analyses of Cu and (right) Ir by the energy-dispersive $\mathrm{x}$-ray spectroscopy for the film sample with $t=30 \mathrm{~nm}$ at $y_{\mathrm{F}}=3 \mathrm{~mm}$

screening of SH materials. SPE is the phenomenon giving rise to the temperature modulation due to the interaction of $\mathbf{J}_{\mathbf{s}}$ and spontaneous magnetization (M). As depicted in Fig. 2a, for the present $\mathrm{Cu}-\mathrm{Ir} / \mathrm{YIG}$ junction, $\mathbf{J}_{\mathrm{s}}$ generated via the SHE of $\mathrm{Cu}-\mathrm{Ir}$ interacts with $\mathbf{M}$ of YIG, which modulates the temperature of the $\mathrm{Cu}-\mathrm{Ir} / \mathrm{YIG}$ junction. According to the previous works on $\mathrm{SPE}^{34,35,39-41}$, the heat current $\left(\mathrm{J}_{\mathrm{q}}\right)$ generated by SPE and the resultant $\Delta T^{\mathrm{SPE}}$ are proportional to $\alpha_{\mathrm{SH}}$. Therefore, by measuring the $\Delta T^{\mathrm{SPE}}$, one can obtain information on the magnitude and sign of the SHE of $\mathrm{Cu}-\mathrm{Ir}$.

The device for the SPE measurement is schematically illustrated in Fig. 2b. The composition-spread film was patterned into the device consisting of two wires with the width of $0.4 \mathrm{~mm}$. One ends of the wires were electrically connected, such that the two wires were connected in series with $\mathbf{J}_{\mathrm{c}}$ flowing along opposite directions. In the device for the SPE measurement, the gradient of composition was formed along the length of $8.0 \mathrm{~mm}$ as shown in Fig. $2 \mathrm{~b}$, and the position in the device was denoted by $y_{\mathrm{D}}$. The infrared radiation thermally emitted from the sample surface was detected while applying an ac $\mathbf{J}_{c}$ with rectangular wave modulation to the $\mathrm{Cu}$-Ir wires. The LIT conditions for the SPE and Joule heating measurements are shown in Fig. $2 c, d$, respectively. The SPE signal was separated from the Joule heating contribution when the first harmonic response of the thermal images was fed without any $\mathrm{dc}$ current offset. For the measurement of Joule heating, on the other hand, the ac $\mathbf{J}_{c}$ together with a dc offset of $J_{c}^{0}$ was applied to the $\mathrm{Cu}$-Ir wires. Although both the SPE and Joule-heating contributions are included in the first harmonic response, the observed signals mainly come from the Joule-heating-induced temperature modulation $\left(\Delta T^{\text {Joule }}\right)$ because $\Delta T^{\text {Joule }}$ is much larger than $\Delta T^{\mathrm{SPE}}$.

First, let us explain the SPE contribution in the $\mathrm{Cu}-\mathrm{Ir}$ wires with $t=30 \mathrm{~nm}$. Figure $2 \mathrm{e}$ shows the amplitude $\left(A^{\mathrm{SPE}}\right)$ and phase $\left(\phi^{\mathrm{SPE}}\right)$ images of SPE-induced temperature modulation at $J_{\mathrm{c}}=10 \mathrm{~mA}$ with the lock-in frequency $(f)$ of $25 \mathrm{~Hz}$. These images were obtained by using the images at the external magnetic field $\left(B_{\text {ext }}\right)$ of 0.1 and $-0.1 \mathrm{~T}$, where $B_{\text {ext }}$ was applied along the in-plane $x$ direction, in order to extract the $B_{\text {ext }}$-odd component. The detailed procedure was reported in ref. 41 , which is effective to subtract the $B$ independent background signals coming from the conventional Peltier effect because the Peltier coefficient of the compositionspread film varies from one position to another. The left and right $\mathrm{Cu}-\mathrm{Ir}$ wires exhibit the opposite phase. Namely, the sign of the temperature modulation is reversed by reversing $J_{c}$. This fact coincides with the symmetry of SPE. The SPE-induced temperature modulation appears only within the wires and the thermal diffusion is suppressed. This is attributable to the dipolar heat sources placed near the sample surface by the $\mathrm{SPE}^{35,39,43}$. Figure $2 \mathrm{~g}$ shows $A^{\mathrm{SPE}}$ as a function of $y_{\mathrm{D}}$ in the left $\mathrm{Cu}-\mathrm{Ir}$ wire with $t=30 \mathrm{~nm}$. $A^{\mathrm{SPE}}$ shows the maximum at $y_{\mathrm{D}} \sim 1.9 \mathrm{~mm}$.

Next, the Joule-heating contribution is examined. Figure $2 \mathrm{f}$ shows the amplitude $\left(A^{\text {Joule }}\right)$ and phase $\left(\phi^{\text {Joule }}\right)$ images of Jouleheating-induced temperature modulation at $J_{\mathrm{c}}{ }^{0}=1 \mathrm{~mA}$ and $\Delta J_{\mathrm{c}}=$ $1 \mathrm{~mA}$ with $f=25 \mathrm{~Hz}$. These images were observed without $B_{\text {ext }}$. Since $A^{\text {Joule }}$ is increased and no sign change of $\phi^{\text {Joule }}$ is observed for the left and right wires, Joule heating increases the temperature of the $\mathrm{Cu}$-Ir wires irrespective of the $\mathbf{J}_{\mathrm{c}}$ direction, which is totally different from the SPE-induced temperature modulation. Similar to the SPE-induced temperature modulation, the spatial variation of $A$ Joule outside the wires is suppressed owing to the high $f$. Therefore, the magnitude of $A^{\text {Joule }}$ provides us the information of local electric conductivity. Figure $2 \mathrm{~h}$ plots $A^{\text {Joule }}$ as a function of $y_{\mathrm{D}}$ in the left $\mathrm{Cu}-\mathrm{Ir}$ wire with $t=30 \mathrm{~nm}$. $A^{\text {Joule }}$ shows the maximum at $y_{\mathrm{D}} \sim 2.1 \mathrm{~mm}$, implying the increase in the resistivity $(\rho)$ of $\mathrm{Cu}-\mathrm{Ir}$ wire at $y_{\mathrm{D}} \sim 2.1 \mathrm{~mm}$. We also evaluated $\rho$ directly using the conventional transport measurement, which is plotted in Fig. 2i. Consequently, Fig. 2h, i revealed the position-dependent $\rho$ values along the $y$ direction of wire. It is noted that $y_{\mathrm{D}} \sim 2.1 \mathrm{~mm}$ exhibiting the maximum $A^{\text {Joule }}$ is the different position from $y_{\mathrm{D}} \sim$ $1.9 \mathrm{~mm}$ for the maximum of $A^{\mathrm{SPE}}$. 
a

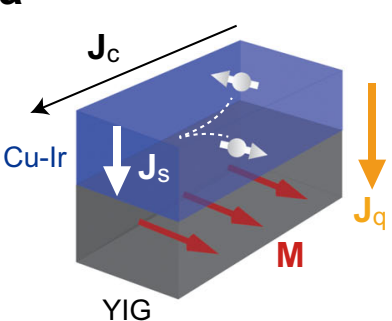

b

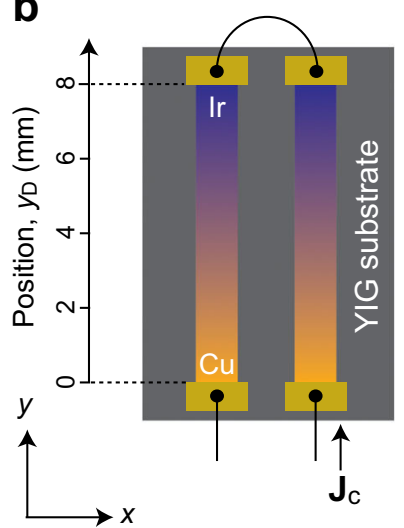

C

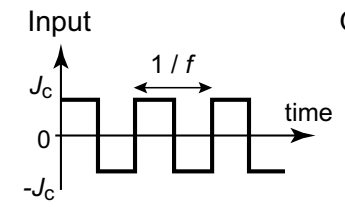

d

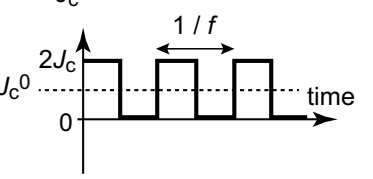

e
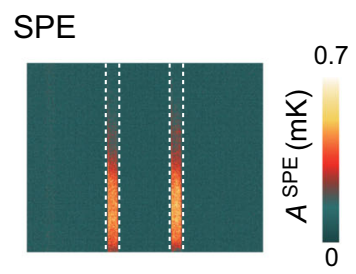

f Joule heating

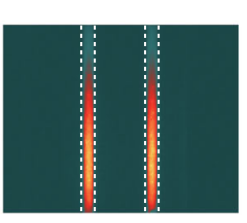

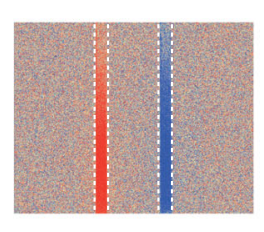

Output
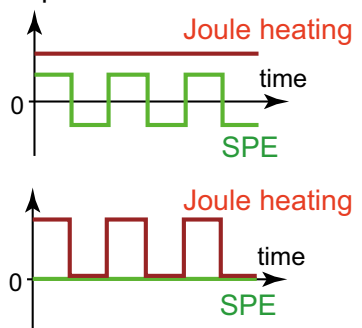

$\vec{E}$
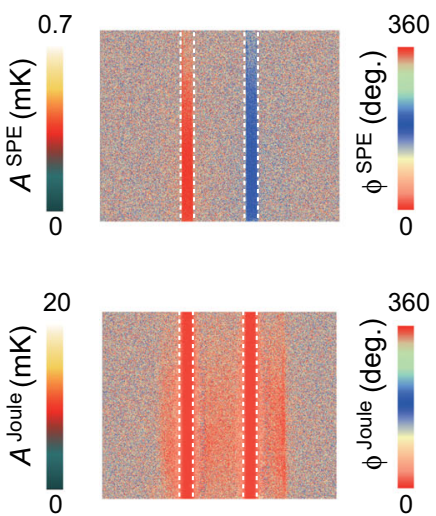
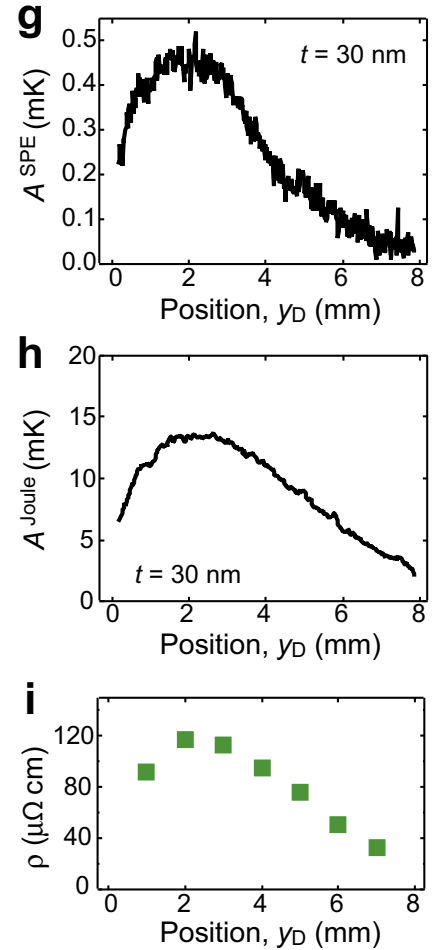

Fig. 2 Thermal imaging of spin Peltier effect. a Concept of the spin-Hall effect (SHE)-induced spin Peltier effect (SPE). For the present Cu-Ir/YIG junction, the SHE in Cu-Ir converts the charge current $\left(\mathbf{J}_{\mathrm{c}}\right)$ into the spin current $\left(\mathbf{J}_{\mathrm{s}}\right)$. The $\mathbf{J}_{\mathrm{s}}$ interacts with magnetization $(\mathbf{M})$ of $\mathrm{YIG}$, which results in the heat current $\left(\mathbf{J}_{\mathrm{q}}\right)$ flow across the Cu-Ir/YIG junction. $\mathbf{b}$ Illustration of device for the SPE measurement, which consists of two wires with the width of 0.4 mm. The position in the device was denoted by $y_{D}$. c Lock-in thermography (LIT) conditions for the SPE and $\mathbf{d}$ Joule heating measurements. e Amplitude ( $A^{\mathrm{SPE}}$ ) and phase $\left(\phi^{\mathrm{SPE}}\right)$ images of SPE-induced temperature modulation for $t=30 \mathrm{~nm}$ at $J_{c}=10 \mathrm{~mA}$ with the lock-in frequency $(f)$ of $25 \mathrm{~Hz}$. These images were obtained by using the images at the external magnetic field $\left(B_{\text {ext }}\right)$ of 0.1 and $-0.1 \mathrm{~T}$, where $B_{\text {ext }}$ was applied along the in-plane $x$ direction, in order to exact the $B_{\text {ext }}$-odd component. $\mathbf{f}$ Amplitude ( $A^{\text {Joule }}$ ) and phase ( $\left.\phi^{\text {Joule }}\right)$ images of Joule-heating-induced temperature modulation for $t=30 \mathrm{~nm}$ at $J_{C}{ }^{0}=$ $1 \mathrm{~mA}$ and $\Delta \mathrm{J}_{\mathrm{c}}=1 \mathrm{~mA}$ with $f=25 \mathrm{~Hz}$. $B_{\text {ext }}$ was not applied. $\mathbf{g} A^{\mathrm{SPE}}$ and $\mathbf{h} A^{\text {Joule }}$ as a function of $y_{\mathrm{D}}$ in the left Cu-Ir wire with $t=30 \mathrm{~nm}$ at $f=25 \mathrm{~Hz}$. These line profiles were obtained by averaging the data points in the $0.2 \mathrm{~mm}$-wide central region of the wire. i Resistivity $(\rho)$ of $\mathrm{Cu}$ - $\mathrm{Ir}$ wire with $t=30 \mathrm{~nm}$ as a function of $y_{D}$.

Spin-Hall effect probed by SPE. The $y_{\mathrm{D}}$ dependence of $A^{\mathrm{SPE}}$ allows us to qualitatively discuss the Ir concentration dependence of $\alpha_{\mathrm{SH}}$ and $\mathrm{SH}$ conductivity $\left(\sigma_{\mathrm{SH}}\right)$ by assuming that the spin mixing conductance at the $\mathrm{Cu}-\mathrm{Ir} / \mathrm{YIG}$ interface and the spin diffusion length in the $\mathrm{Cu}-\mathrm{Ir}$ do not show a remarkable position dependence. $\Delta T^{\mathrm{SPE}} / j_{\mathrm{c}}$ and $\Delta T^{\mathrm{SPE}} / E$ are proportional to $\alpha_{\mathrm{SH}}$ and $\sigma_{\mathrm{SH}}\left(=\alpha_{\mathrm{SH}} \sigma_{\mathrm{Cu}-\mathrm{Ir}}\right)$, respectively, where $E$ is the electric field and $\sigma_{\mathrm{Cu}-\mathrm{Ir}}$ is the conductivity of $\mathrm{Cu}-\mathrm{Ir} . \Delta T^{\mathrm{SPE}}$ is given by the real part of the complex temperature modulation output by the LIT: $\Delta T^{\mathrm{SPE}}=A^{\mathrm{SPE}} \cos \left(\phi^{\mathrm{SPE}}\right)$, because the phase delay due to thermal diffusion in the SPE signal is negligibly small 135,39 .

Figure $3 \mathrm{a}$, b show the $\Delta T^{\mathrm{SPE}} / j_{\mathrm{c}}$ and $\Delta T^{\mathrm{SPE}} / E$ as a function of $x_{\mathrm{Ir}}$ for $t=10,20$, and $30 \mathrm{~nm}$, where $x_{\text {Ir }}$ was estimated using the position dependence of $x_{\mathrm{Ir}}$ shown in Fig. $1 \mathrm{~b} . \Delta T^{\mathrm{SPE}} / j_{\mathrm{c}}$ exhibits the maximum value around $x_{\mathrm{Ir}}=25$ at.\% while $\Delta \mathrm{T}^{\mathrm{SPE}} / \mathrm{E}$ is monotonically decreased as $x_{\text {Ir }}$ is increased. These results suggest that $\alpha_{\mathrm{SH}}$ is enhanced around $x_{\mathrm{Ir}}=25 \mathrm{at} . \%$. Figure $3 \mathrm{c}$ is the plot of $t$ dependence of $\Delta T^{\mathrm{SPE}} / j_{\mathrm{c}}$ for several Ir concentrations. $t=20 \mathrm{~nm}$ shows a larger $\Delta T^{\mathrm{SPE}} / j_{\mathrm{c}}$ than those for $t=10$ and $30 \mathrm{~nm}$. We have no clear reason for this $t$ dependence at present.

In order to elucidate the mechanism of the enhanced $\alpha_{\mathrm{SH}}$ when $x_{\mathrm{Ir}}$ is increased up to 25 at. $\%, \Delta T^{\mathrm{SPE}} / E$ versus $\sigma_{\mathrm{Cu}-\mathrm{Ir}}$ is summarized in Fig. 3d, where the data in the Ir concentration range from 0 to 25 at.\% (hatched areas in Fig. 3a, b) were plotted for $t=10,20$, and $30 \mathrm{~nm}$. This plot corresponds to $\sigma_{\mathrm{SH}}$ versus $\sigma_{\mathrm{Cu}-\mathrm{Ir}}$. One can see that $\sigma_{\mathrm{SH}}$ remains to be almost constant regardless of $\sigma_{\mathrm{Cu}-\mathrm{Ir}}$. This tendency implies that either the side jump process or the intrinsic mechanism due to the Berry curvature is a dominant mechanism for the SHE in the $\mathrm{Cu}$-Ir. The details will be discussed later. An important finding is the enhanced $\alpha_{\mathrm{SH}}$ around $x_{\mathrm{Ir}}=25$ at.\% that is definitely out of the solubility limit in the $\mathrm{Cu}$-rich region of bulk phase diagram ${ }^{33}$.

Quantitative analysis of spin-Hall angle. Here we evaluate the SH efficiency for the $\mathrm{Cu}_{76} \mathrm{Ir}_{24}$ alloy, which is in the composition range showing the enhanced $\alpha_{\mathrm{SH}}$, using the harmonic Hall voltage measurement ${ }^{44-48}$. For this quantitative measurement, we prepared a thin film consisting of $\mathrm{Cu}_{76} \mathrm{Ir}_{24}(t \mathrm{~nm})|\mathrm{CoFeB}(2 \mathrm{~nm})| \mathrm{Al}$ $(2 \mathrm{~nm})$ on the sapphire $c$-plane substrate, where the $\mathrm{Cu}_{76} \mathrm{Ir}_{24}$ is a wedge-shaped layer with $t$ in the range from 1 to $19 \mathrm{~nm}$, and the $\mathrm{CoFeB}$ is in-plane magnetized. The thin film was patterned into the Hall bar shape as illustrated in Fig. 4a. The in-phase first harmonic $\left(V^{\omega}\right)$ and the out-of-phase second harmonic voltages $\left(V^{2 \omega}\right)$ were detected using two lock-in amplifiers under the application of ac current with the frequency of $\omega / 2 \pi=172.1 \mathrm{~Hz}$, allowing us to separately determine two kinds of torques acting on the CoFeB magnetic moment (m). In general, SHE gives rise to the damping-like (DL) torque pointing along $\mathbf{m} \times(\mathbf{m} \times \mathbf{s})$ with a magnitude of $B_{\mathrm{DL}}$. Another torque mainly coming from the Rashba-Edelstein effect (REE) is called the field-like (FL) torque directed along $\mathbf{m} \times \mathbf{s}$ with a magnitude of $B_{\mathrm{FL}}$. By considering the dependence of $V^{\omega}$ and $V^{2 \omega}$ on the in-plane field angle $(\theta$, depicted 

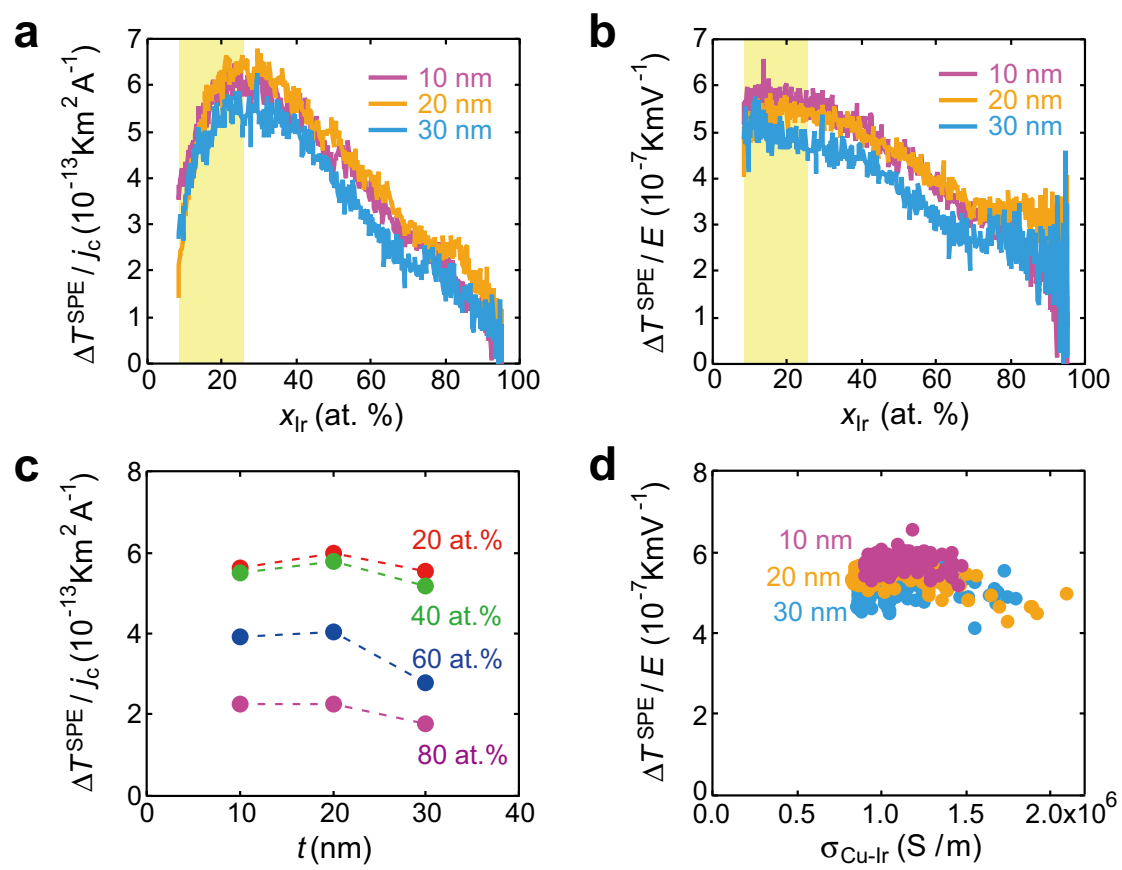

Fig. 3 Composition and thickness dependence of spin-Hall effect in Cu-Ir. a $\Delta T^{S P E} / j_{c}$ and $\mathbf{b} \Delta T \mathrm{SPE} / E$ as a function of $x_{\mathrm{Ir}}$ for $t=10,20$, and $30 \mathrm{~nm}$, where $j_{\mathrm{c}}$ and $E$ are the current density and the electric field. $\mathbf{c} t$ dependence of $\Delta T S P E / j_{c}$ for different Ir concentrations. $\mathbf{d} \Delta T^{S P E} / E$ versus $\sigma_{\mathrm{Cu}-\mathrm{Ir}}$ for $t=10,20$, and $30 \mathrm{~nm}$, where $\sigma_{\mathrm{Cu}-\mathrm{Ir}}$ is the conductivity of $\mathrm{Cu}-\mathrm{Ir}$.

in Fig. 4a), the following expressions of $R^{\omega}$ and $R^{2 \omega}$ for the Hall resistance are obtained ${ }^{47,48}$.

$$
\begin{gathered}
R^{\omega}=R_{\mathrm{PHE}} \sin 2 \theta \\
R^{2 \omega}=-\left(R_{\mathrm{AHE}} \frac{B_{\mathrm{DL}}}{B_{\mathrm{ext}}+B_{\mathrm{ani}}}+R_{\nabla T}^{0}\right) \cos \theta \\
+2 R_{\mathrm{PHE}}\left(\frac{B_{\mathrm{FL}}+B_{\mathrm{Oe}}}{B_{\mathrm{ext}}}\right) \cos 2 \theta \cos \theta,
\end{gathered}
$$

where $R_{\mathrm{PHE}}$ and $R_{\mathrm{AHE}}$ are the resistance changes originating from the planar Hall effect (PHE) and the anomalous Hall effect (AHE), respectively. $B_{\mathrm{Oe}}$ and $B_{\text {ani }}$ are the Oersted field due to the current flow and the anisotropy field, respectively. $R_{\nabla T}^{0}$ denotes the second harmonic Hall signal due to thermoelectric effects including the anomalous Nernst effect and spin Seebeck effect. Using the coefficients of $C$ and $D$, Eq. (3) can be rewritten as

$$
R^{2 \omega}=C \cos \theta+D \cos 2 \theta \cos \theta \text {. }
$$

Therefore, the measurement of in-plane field angular dependence enables us to separate the DL (mainly of SHE origin) contribution with thermoelectric effects and the FL (mainly of REE origin) contribution. Furthermore, DL contribution and the thermoelectric effects can be separated by investigating the $B_{\text {ext }}$ dependence.

Figure $4 \mathrm{~b}, \mathrm{c}$ show the $\theta$ dependence of $R^{\omega}$ and $R^{2 \omega}$, respectively, for $t=5.8 \mathrm{~nm}$ with $B_{\text {ext }}=0.1 \mathrm{~T}$ at $300 \mathrm{~K}$. From the $\theta$ dependence of $R^{\omega}$ (Fig. 4b), we obtained $R_{\mathrm{PHE}}=-0.09 \mathrm{~m} \Omega$. In Fig. $4 \mathrm{c}$, the orange open circles denote the measured data, and the blue curve is the result of fitting by $\cos \theta$. The green open circles represent the values subtracting the $\cos \theta \operatorname{component}$ from the measured data, and the black curve is the result of fitting by the $\cos 2 \theta \cos \theta$ function. As seen in Fig. $4 \mathrm{c}$, the $\theta$ dependence of $R^{2 \omega}$ is fitted by Eq. (4). We also measured the AHE with $B_{\text {ext }}$ applied normal to the device plane (Fig. $4 \mathrm{~d}$ ), and $R_{\mathrm{AHE}}=1.74 \Omega$ and
$B_{\text {ani }}=0.9 \mathrm{~T}$ were obtained. The coefficient $C(D)$ as a function of the inverse of $B_{\text {ext }}+B_{\text {ani }}$ (the inverse of $B_{\text {ext }}$ ) is plotted in Fig. $4 \mathrm{e}$ (Fig. 4f), where the large $B_{\text {ext }}$ was applied to do the correct estimation of the thermo-electric contribution. From the linear fits, $B_{\mathrm{DL}}$ and $B_{\mathrm{FL}}$ are evaluated to be 0.74 and $0.33 \mathrm{mT}$, respectively. According to the previous papers ${ }^{47,48}$, the DL torque efficiency $\left(\xi_{\mathrm{DL}}\right)$ and the FL torque efficiency $\left(\xi_{\mathrm{FL}}\right)$ are given by $\xi_{\mathrm{DL}}=\frac{2 e}{\hbar} \frac{B_{\mathrm{DL}} M_{\mathrm{s}} t_{\mathrm{CPB}}}{j_{\mathrm{Cu}-\mathrm{Ir}}}$ and $\xi_{\mathrm{FL}}=\frac{2 e}{\hbar} \frac{B_{\mathrm{FL}} M_{\mathrm{s}} t_{\mathrm{CFB}}}{j_{\mathrm{Cu}-\mathrm{Ir}}}$, where $M_{\mathrm{s}}$ is the saturation magnetization of $\mathrm{CoFeB}$, which was experimentally obtained to be $800 \mathrm{kA} \mathrm{m}^{-1}, t_{\mathrm{CFB}}$ is the CoFeB layer thickness, and $j_{\mathrm{Cu}-\mathrm{Ir}}$ is the current density flowing in the $\mathrm{Cu}-\mathrm{Ir}$ layer. With the parameters of $M_{\mathrm{s}}=800 \mathrm{kA} \mathrm{m}^{-1}$ and $j_{\mathrm{Cu}-\mathrm{Ir}}=8.1 \times 10^{6} \mathrm{~A} \mathrm{~cm}^{-2}, \xi_{\mathrm{DL}}=4.5 \%$ and $\xi_{\mathrm{FL}}=2.0 \%$ were obtained for $t=5.8 \mathrm{~nm}$. The accuracy in the estimation of $\xi_{\mathrm{FL}}$ should be noted here. The small $\cos 2 \theta \cos \theta$ dependence in $R^{2 \omega}$, which is attributed to the small $R_{\mathrm{PHE}}$, gives rise to the low accuracy for the estimation of $\xi_{\mathrm{FL}}$. Also, one is aware of the apparent parabolic $1 / B_{\text {ext }}$ dependence of coefficient $D$ in Fig. 4f. This is attributable to the unwanted cross talk between $C$ and $D$ in the fitting and a small imperfection in $C$, which is probably due to the slight misalignment or the experimental uncertainty, leads to a large relative error in $D$. This is another source of the low accuracy for the estimation of $\xi_{\mathrm{FL}}$. In contrast to $\xi_{\mathrm{FL}}$, the estimation of $\xi_{\mathrm{DL}}$ is not affected by the magnitude of $R_{\mathrm{PHE}}$. Hereafter, we will focus on the DL torque component, and evaluate $\alpha_{\mathrm{SH}}$ for the $\mathrm{Cu}_{76} \mathrm{Ir}_{24}$ from $\xi_{\mathrm{DL}}$.

According to ref. ${ }^{49}$, with the assumption that the interface spin transparency is $<1$ and the spin backflow is dominant at the wellordered interface, the $t$ dependence of $\xi_{\mathrm{DL}}$ per unit applied electric field, $\xi_{\mathrm{DL}}^{\mathrm{E}}=\xi_{\mathrm{DL}} / \rho$, is expressed as

$$
\xi_{\mathrm{DL}}^{\mathrm{E}}(t)=\frac{2 e}{\hbar} \sigma_{\mathrm{SH}}\left[1-\operatorname{sech}\left(t / \lambda_{\mathrm{SD}}\right)\right]\left(1+\frac{\tanh \left(t / \lambda_{\mathrm{SD}}\right)}{2 \lambda_{\mathrm{SD}} \rho \operatorname{Re}\left[G_{\mathrm{MIX}}\right]}\right)^{-1},
$$

where $\lambda_{\mathrm{SD}}$ is the spin diffusion length of $\mathrm{Cu}_{76} \mathrm{Ir}_{24}$ and $\operatorname{Re}\left[G_{\mathrm{MIX}}\right]$ is the real part of the spin mixing conductance. Using the $t$ dependence of $\rho$ (Fig. $4 \mathrm{~g}$ ), $\xi_{\mathrm{DL}}^{\mathrm{E}}$ is plotted as a function of $t$ in 

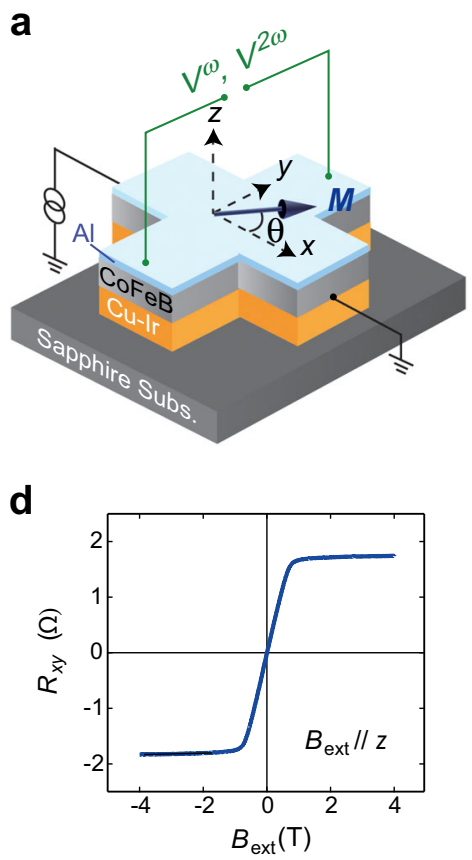

b

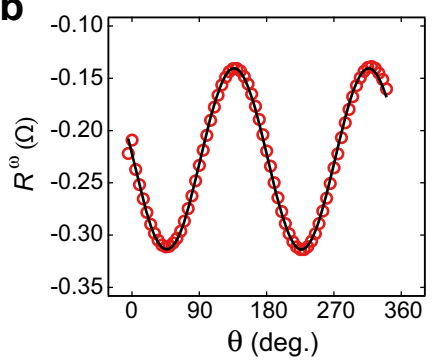

e

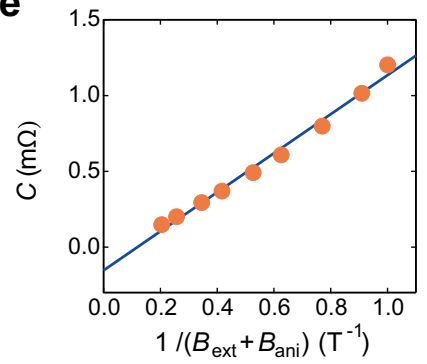

g

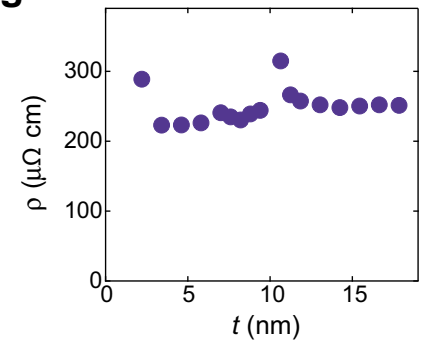

C

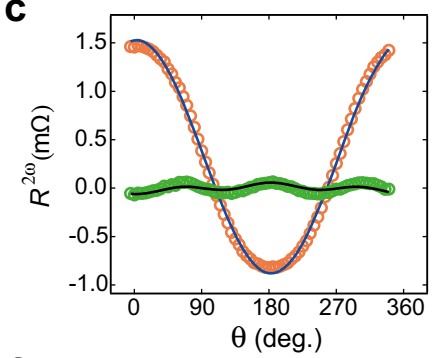

f

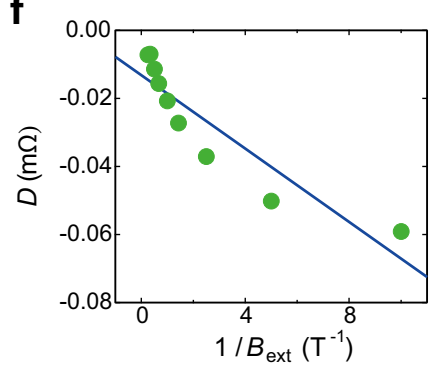

h

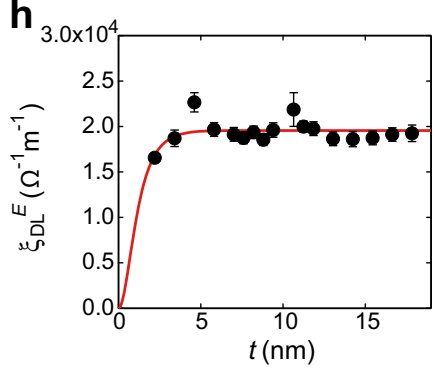

Fig. 4 Evaluation of spin-Hall effect using harmonic Hall voltage. a Schematic illustration of Hall bar-shaped device together with the setup of harmonic Hall voltage measurement, where the stacking structure of thin film is $\mathrm{Cu}_{76} \mathrm{Ir}_{24}(t \mathrm{~nm})|\mathrm{CoFeB}(2 \mathrm{~nm})| \mathrm{Al}(2 \mathrm{~nm})$ on the sapphire c-plane substrate. The inplane field angle $(\theta)$ was rotated. $\mathbf{b} \theta$ dependence of $R^{\omega}$ and $\mathbf{c} R^{2 \omega}$ for $t=5.8 \mathrm{~nm}$ with $B_{\text {ext }}=0.1 \mathrm{~T}$ at $300 \mathrm{~K}$. In $\mathbf{b}$, the red open circles denote the measured data, and the black solid curve represents the result of fitting. In $\mathbf{c}$, the orange open circles denote the measured data, and the blue curve is the result of fitting by $\cos \theta$. The green open circles represent the values subtracting the $\cos \theta \operatorname{component}$ from the measured data, and the black curve is the result of fitting by the $\cos 2 \theta \cos \theta$ function. d Anomalous Hall loop with $B_{\text {ext }}$ applied normal to the device plane for $t=5.8 \mathrm{~nm}$. e Coefficients $C$ and $\mathbf{f} D$ of second harmonic Hall resistance $\left(R^{2 \omega}\right)$ as a function of the inverse of $B_{\text {ext }}+B_{\text {ani }}$ and the inverse of $B_{\text {ext }}$, respectively for $t=5.8 \mathrm{~nm}$, where $B_{\text {ani }}$ is the anisotropy field. The solid lines are the results of fitting. $\mathbf{g} t$ dependence of the resistivity $(\rho)$ of the $\mathrm{Cu}_{76} \mathrm{Ir}_{24}$. $\mathbf{h}$ Damping-like torque efficiency per unit applied electric field $\left(\xi_{D L}^{E}\right)$ as a function of $t$, where the solid line is the result of fitting by Eq. (5). The error bars come from the standard deviation obtained by the linear fit in $\mathbf{e}$.

Fig. 4h. From the fit to the data with Eq. (5) and the parameters of $\rho=261 \mu \Omega \mathrm{cm}$ and $\operatorname{Re}\left[G_{\mathrm{MIX}}\right]=1 \times 10^{15} \Omega^{-1} \mathrm{~m}^{-2}, \sigma_{\mathrm{SH}}$ and $\lambda_{\mathrm{SD}}$ of the $\mathrm{Cu}_{76} \mathrm{Ir}_{24}$ were estimated to be $(2.41 \pm 0.07) \times 10^{4}[\hbar / 2 e]$ $\Omega^{-1} \mathrm{~m}^{-1}$ and $0.83 \pm 0.14 \mathrm{~nm}$, respectively. Finally, we obtained $\alpha_{\mathrm{SH}}=6.29 \pm 0.19 \%$ using the harmonic Hall voltages. In addition to the harmonic Hall voltage measurement, we carried out the spin-Hall magnetoresistance measurement (see Supplementary Note 1 and Supplementary Fig. 1), and estimated $\alpha_{\mathrm{SH}}=$ $6.95 \pm 0.05 \%$ and $\lambda_{\mathrm{SD}}=1.67 \pm 0.04 \mathrm{~nm}$. These values are close to the values obtained by the harmonic Hall voltage measurement. Then, we conclude that the $\mathrm{Cu}_{76} \mathrm{Ir}_{24}$ exhibits large $\alpha_{\mathrm{SH}}>6 \%$.

Since $\mathrm{Bi}$-doped $\mathrm{Cu}$ is believed to be a good $\mathrm{SH}$ material ${ }^{25}$, we performed the same LIT-based SPE measurements using a $\mathrm{Cu}-\mathrm{Bi}$ composition-spread film on the YIG substrate. In contrast to the $\mathrm{Cu}-\mathrm{Ir}$, the non-equilibrium $\mathrm{Cu}-\mathrm{Bi}$ binary alloys do not show remarkable SHE (see Supplementary Fig. 2). According to ref. ${ }^{25}$, doping a very small amount of $\mathrm{Bi}$ is effective to obtain the large SHE. On the other hand, this study examined the effect of alloying on $\mathrm{SHE}$ for the $\mathrm{Cu}-\mathrm{Bi}$ over a wide composition range, and do not examine on the $\mathrm{Cu}$-rich region with a very small amount of $\mathrm{Bi}$. This is a possible reason why the present $\mathrm{Cu}-\mathrm{Bi}$ does not exhibit the definite SHE. As a result, we found that the alloying is not effective for the $\mathrm{Cu}-\mathrm{Bi}$ binary alloys.

\section{Discussion}

This work has three major achievements: (i) development of nonequilibrium $\mathrm{Cu}$-Ir binary alloys beyond the solubility limit by the sputtering method, (ii) finding that the non-equilibrium $\mathrm{Cu}-\mathrm{Ir}$ exhibits the large SHE although neither $\mathrm{Cu}$ nor $\mathrm{Ir}$ exhibits remarkable SHE, and (iii) elucidation of mechanism of SHE for the non-equilibrium $\mathrm{Cu}$-Ir. Our comprehensive investigation revealed that the value of $\alpha_{\mathrm{SH}}$ shows the maximum around $x_{\mathrm{Ir}}=$ 25 at.\%, which is much larger than the solubility limit. Even at such a non-equilibrium composition, we successfully obtained the single-phase $\mathrm{Cu}$-Ir binary alloy. $\alpha_{\mathrm{SH}}>6 \%$ obtained for $\mathrm{Cu}_{76} \mathrm{Ir}_{24}$ is comparable to or larger than that for $\mathrm{Pt}^{5}$.

Finally, we complement the mechanism of SHE for the nonequilibrium $\mathrm{Cu}-\mathrm{Ir}$ binary alloy. As shown in Fig. $3 \mathrm{~d}$, the plot of $\Delta T^{\mathrm{SPE}} / E$ versus $\sigma_{\mathrm{Cu}-\mathrm{Ir}}$ suggests that $\sigma_{\mathrm{SH}}$ keeps almost constant regardless of $\sigma_{\mathrm{Cu}-\mathrm{Ir}}$. This indicates that either the side jump process or the intrinsic mechanism due to the Berry curvature is a dominant mechanism for the SHE in the $\mathrm{Cu}-\mathrm{Ir}$. It is worth noting 
that the previous work for the $\mathrm{Ir}$-doped $\mathrm{Cu}$ reported that the skew scattering plays the dominant role for the SHE in the Ir-doped $\mathrm{Cu}$ with $x_{\mathrm{Ir}}<12$ at. $\%^{20}$. Therefore, the present non-equilibrium $\mathrm{Cu}-\mathrm{Ir}$ binary alloy exhibits the SHE totally different from the Ir-doped $\mathrm{Cu}$ quantitatively and qualitatively. Our discovery suggests that a non-equilibrium alloy consisting of nonmagnets with negligible SHE has a potential as an SH material, and opens a new direction for the exploration of $\mathrm{SH}$ materials.

\section{Methods}

Fabrication of composition-spread $\mathrm{Cu}-\mathrm{Ir}$ (Cu-Bi) film. The $\mathrm{Cu}-\mathrm{Ir}(\mathrm{Cu}-\mathrm{Bi})$ composition-spread films were fabricated on a YIG substrate at ambient temperature using DC magnetron sputtering with a base pressure of $<6.0 \times 10^{-6} \mathrm{~Pa}$ and a process Ar gas pressure of $0.4 \mathrm{~Pa}$. The YIG substrate consists of a $23-\mu \mathrm{m}-$ thick single-crystalline YIG (111) film uniformly grown on a single-crystalline $\mathrm{Gd}_{3} \mathrm{Ga}_{5} \mathrm{O}_{12}$ (111) substrate by means of a liquid phase epitaxy method. To improve the lattice matching between YIG and $\mathrm{Gd}_{3} \mathrm{Ga}_{5} \mathrm{O}_{12}$, a tiny amount of Y in YIG is substituted by Bi. The substrate was cut into a $10 \times 10 \mathrm{~mm}^{2}$ square shape. As described in the main text, the $\mathrm{Cu}-\mathrm{Ir}$ composition-spread films were prepared by repeating the following three processes: (1) deposition of a wedge-shaped $\mathrm{Cu}$ layer using the linear moving shutter, (2) rotation of the substrate by $180^{\circ}$, and (3) deposition of a wedge-shaped Ir layer using the linear moving shutter, where the total thickness of a Cu/Ir pair was designed to be $0.5 \mathrm{~nm}$. The deposition of $0.5 \mathrm{~nm}$ thick $\mathrm{Cu} / \mathrm{Ir}$ pair was repeated 20 times, 40 times, and 60 times for the samples with total thicknesses of 10, 20, and $30 \mathrm{~nm}$, respectively. All the layers were deposited at room temperature in order to prevent from the appearance of thermodynamically stable phase. The same deposition procedure was used for the $\mathrm{Cu}-\mathrm{Bi}$ compositionspread films. The $\mathrm{Cu}(\mathrm{Ir})$ layers were deposited at a rate of $0.052 \mathrm{~nm} \mathrm{~s}^{-1}(0.034 \mathrm{~nm}$ $\mathrm{s}^{-1}$ ). For the LIT measurement, the composition-spread film was patterned into a rectangular shape with a size of $8.0 \mathrm{~mm} \times 0.4 \mathrm{~mm}$ by sputtering the layers through a metallic shadow mask, where the composition gradient is along the $8 \mathrm{~mm}$ direction. All the films were capped with a 2 -nm-thick $\mathrm{Al}$ film to prevent oxidation.

In addition to the composition-spread films, for the harmonic Hall voltage measurement, we prepared the thin film consisting of $\mathrm{Cu}_{76} \mathrm{Ir}_{24}(t \mathrm{~nm}) \mid \mathrm{CoFeB}$ $(2 \mathrm{~nm}) \mid \mathrm{Al}(2 \mathrm{~nm})$ on the sapphire $c$-plane substrate. The thickness of wedge-shaped $\mathrm{Cu}_{76} \mathrm{Ir}_{24}$ layer was continuously varied from 1 to $19 \mathrm{~nm}$ in the lateral length of $20 \mathrm{~mm}$, where the $\mathrm{Cu}_{76} \mathrm{Ir}_{24}$ layer was formed by co-sputtering of $\mathrm{Cu}$ and Ir. We experimentally confirmed that the DL torque efficiencies were almost the same for the films prepared by alternate layer deposition method and the co-sputtering method, indicating that there is no difference in the properties between the composition-spread films and the films for the harmonic Hall voltage measurement. The composition of $\mathrm{Cu}_{76} \mathrm{Ir}_{24}$ was determined by the inductively coupled plasma atomic emission spectroscopy. The thin film was patterned into the Hall bar shape through the use of photo lithography and Ar ion milling.

Structural characterization and magnetization measurement. For structural analysis of the composition-spread films, XRD was measured with a Rigaku SmartLab XRD diffractometer, using $\mathrm{Cu}-\mathrm{Ka}_{1}$ radiation $(\lambda=0.15406 \mathrm{~nm})$. The $\mathrm{x}$ ray was incident on the sample in the Bragg-Brentano geometry as a parallel beam through a length limit slit of $0.5 \mathrm{~mm}$ in the direction perpendicular to the composition gradient and detected in a 2D detector (Rigaku PILATUS100K/R). The measurement was performed at different positions on the films along the composition gradient at an interval of $1 \mathrm{~mm}$. The $\mathrm{Ir}(\mathrm{Bi})$ concentration of the $\mathrm{Cu}-\mathrm{Ir}$ (Cu-Bi) composition-spread film was measured by EPMA (JEOL, JXA-8530F). The transmission electron microscope observation was carried out by using JEOL, JEMARM200F. The magnetic properties of the CoFeB layer were characterized using the vibrating sample magnetometer at room temperature.

LIT measurements. In the SPE and Joule-heating measurements based on the LIT, we applied a square-wave-modulated AC charge current $(f=25 \mathrm{~Hz})$ with $J_{\mathrm{c}}$ to the $\mathrm{Cu}-\mathrm{Ir}(\mathrm{Cu}-\mathrm{Bi})$ layer and extracted thermal images oscillating with the same $f$. The first harmonic contribution of the obtained thermal images was transformed into the $A$ and $\phi$ images through Fourier analysis. The $A$ image shows the distribution of the magnitude of the temperature modulation generated by the SPE or Joule heating. The $\phi$ image shows the sign of the temperature modulation in the SPE measurements, while it gives the information about the time delay due to thermal diffusion in the Joule-heating measurements. In the SPE (Joule-heating) measurements, the DC offset of the square-wave-modulated AC charge current is zero $\left(J_{c} / 2\right)$. To enhance infrared emissivity, the surface of the samples was coated with insulating black ink with the high emissivity $(>0.95)$, commercially available from Japan Sensor Corporation. The infrared radiation intensity detected by an infrared camera is converted into temperature information by the calibration method shown in ref. ${ }^{50}$. All the LIT observations were carried out at room temperature and atmospheric pressure.

Harmonic Hall voltage measurement. The measurement was performed in a physical property measurement system (PPMS) equipped with horizontal rotator option. The device under test (a Hall bar) was wire-bonded on a standard holder designed for performing field scans within the azimuthal plane. A sinusoidal signal of amplitude $1 \mathrm{~mA}$ for the device with $t=2.2 \mathrm{~nm}$ and $5 \mathrm{~mA}$ for other devices and of frequency $13.7 \mathrm{~Hz}$ was applied using a Keithley $6221 \mathrm{DC}$ and AC current source meter. The first and second harmonic Hall resistances were simultaneously measured by two NF LI5660 lock-in amplifiers.

\section{Data availability}

The data that support the findings of this study are available from the corresponding authors upon reasonable request.

Received: 18 June 2020; Accepted: 21 September 2020; Published online: 14 October 2020

\section{References}

1. Dyakonov, M. I. \& Perel, V. I. Current-induced spin orientation of electrons in semiconductors. Phys. Lett. A 35, 459-460 (1971).

2. Hirsch, J. E. Spin Hall effect. Phys. Rev. Lett. 83, 1834-1837 (1999).

3. Kato, Y. K., Myers, R. C., Gossard, A. C. \& Awschalom, D. D. Observation of the spin Hall effect in semiconductors. Science 306, 1910-1913 (2004).

4. Wunderlich, J., Kaestner, B., Sinova, J. \& Jungwirth, T. Experimental observation of the spin-Hall effect in a two-dimensional spin-orbit coupled semiconductor system. Phys. Rev. Lett. 94, 047204 (2005).

5. Hoffman, A. Spin Hall effects in metals. IEEE Trans. Magn. 49, 5172-5192 (2013)

6. Mellnik, A. R. et al. Spin-transfer torque generated by a topological insulator Nature 511, 449-451 (2014).

7. Fan, Y. et al. Magnetization switching through giant spin-orbit torque in a magnetically doped topological insulator heterostructure. Nat. Mater. 13, 699-704 (2014).

8. Miao, B. F., Huang, S. Y., Qu, D. \& Chen, C. L. Inverse spin Hall effect in a ferromagnetic metal. Phys. Rev. Lett. 111, 066602 (2013).

9. Wu, S. M., Hoffman, J., Pearson, J. E. \& Bhattacharya, A. Unambiguous separation of the inverse spin Hall and anomalous Nernst effects within a ferromagnetic metal using the spin Seebeck effect. Appl. Phys. Lett. 105, 092409 (2014)

10. Seki, T. et al. Observation of inverse spin Hall effect in ferromagnetic FePt alloys using spin Seebeck effect. Appl. Phys. Lett. 107, 092401 (2015).

11. Gibbons, J. D., MacNeill, D., Buhrman, R. A. \& Ralph, D. C. Reorientable spin direction for spin current produced by the anomalous Hall effect. Phys. Rev. Appl. 9, 064033 (2018).

12. Iihama, S. et al. Spin-transfer torque induced by the spin anomalous Hall effect. Nat. Electron. 1, 120-123 (2018)

13. Seki, T., Iihama, S., Taniguchi, T. \& Takanashi, K. Large spin anomalous Hall effect in $L 1_{0}$-FePt: symmetry and magnetization switching. Phys. Rev. B 100, 144427 (2019)

14. Saitoh, E., Ueda, M., Miyajima, H. \& Tatara, G. Conversion of spin current into charge current at room temperature: inverse spin-Hall effect. Appl. Phys. Lett. 88, 182509 (2006).

15. Kimura, T., Otani, Y., Sato, T., Takahashi, S. \& Maekawa, S. Roomtemperature reversible spin Hall effect. Phys. Rev. Lett. 98, 156601 (2007).

16. Liu, L., Moriyama, T., Ralph, D. C. \& Buhrman, R. A. Spin-torque ferromagnetic resonance induced by the spin Hall effect. Phys. Rev. Lett. 106, 36601 (2011).

17. Liu, L. et al. Spin-torque switching with the giant spin Hall effect of tantalum. Science 336, 555 (2012).

18. Kim, J., Sheng, P., Takahashi, S., Mitani, S. \& Hayashi, M. Spin Hall magnetoresistance in metallic bilayers. Phys. Rev. Lett. 116, 097201 (2016)

19. Pai, C.-F. et al. Spin transfer torque devices utilizing the giant spin Hall effect of tungsten. Appl. Phys. Lett. 101, 122404 (2012).

20. Niimi, Y. et al. Extrinsic spin Hall effect induced by iridium impurities in copper. Phys. Rev. Lett. 106, 126601 (2011).

21. Yamanouchi, M. et al. Three terminal magnetic tunnel junction utilizing the spin Hall effect of iridium-doped copper. Appl. Phys. Lett. 102, 212408 (2013).

22. Fert, A. \& Levy, P. M. Spin Hall effect induced by resonant scattering on impurities in metals. Phys. Rev. Lett. 106, 157208 (2011).

23. Cramer, J. et al. Complex terahertz and direct current inverse spin Hall effect in YIG/ $\mathrm{Cu}_{1-\mathrm{x}} \mathrm{I} \mathrm{r}_{\mathrm{x}}$ bilayers across a wide concentration range. Nano Lett. $\mathbf{1 8}$, 1064-1069 (2018)

24. Masuda, H., Seki, T., Lau, Y.-C., Kubota, T. \& Takanashi, K. Interlayer exchange coupling through $\mathrm{Ir}$-doped $\mathrm{Cu}$ spin Hall material. Phys. Rev. B 101, 224413-1-10 (2020)

25. Niimi, Y. et al. Giant spin Hall effect induced by skew scattering from bismuth impurities inside thin film CuBi alloys. Phys. Rev. Lett. 109, 156602 (2012). 
26. Ramaswamy, R. et al. Extrinsic spin Hall effect in $\mathrm{Cu}_{1-x} \mathrm{Pt}_{x}$. Phys. Rev. Appl. 8 , 024034 (2017).

27. Tian, K. \& Tiwari, A. CuPt alloy thin films for application in spin thermoelectrics. Sci. Rep. 9, 3133 (2019).

28. Wong, G. D. H. et al. Thermal behavior of spin-current generation in $\mathrm{Pt}_{x} \mathrm{Cu}_{1-\mathrm{x}}$ devices characterized through spin-torque ferromagnetic resonance. Sci. Rep. 10, 9631 (2020).

29. Gu, B. et al. Surface-assisted spin Hall effect in Au films with Pt impurities. Phys. Rev. Lett. 105, 216401 (2010).

30. Obstbaum, M. et al. Tuning spin Hall angles by alloying. Phys. Rev. Lett. 117, 167204 (2016)

31. Laczkowski, P. et al. Experimental evidences of a large extrinsic spin Hall effect in AuW alloy. Appl. Phys. Lett. 104, 142403 (2014).

32. Ishikuro, Y., Kawaguchi, M., Kato, N., Lau, Y.-C. \& Hayashi, M. Dzyaloshinskii-Moriya interaction and spin-orbit torque at the Ir/Co interface. Phys. Rev. B 99, 134421 (2019).

33. Chakrabarti, D. J. \& Laughlin, D. E. in Cu-Ir (Copper-Iridium), Binary Alloy Phase Diagrams 2nd edn, Vol. 2 (ed. Massalski, T. B.) 1426-1427 (1990).

34. Flipse, J. et al. Observation of the spin Peltier effect for magnetic insulators Phys. Rev. Lett. 113, 027601 (2014).

35. Daimon, S., Iguchi, R., Hioki, T., Saitoh, E. \& Uchida, K. Thermal imaging of spin Peltier effect. Nat. Commun. 7, 13754 (2016).

36. Straube, H., Wagner, J.-M. \& Breitenstein, O. Measurement of the Peltier coefficient of semiconductors by lock-in thermography. Appl. Phys. Lett. 95, 052107 (2009)

37. Breitenstein, O., Warta, W. \& Langenkamp, M. Lock-in Thermography: Basics and Use for Evaluating Electronic Devices and Materials (Springer, Berlin and Heidelberg, 2010).

38. Wid, O. et al. Investigation of the unidirectional spin heat conveyer effect in a $200 \mathrm{~nm}$ thin yttrium iron garnet film. Sci. Rep. 6, 28233 (2016).

39. Daimon, S., Uchida, K., Iguchi, R., Hioki, T. \& Saitoh, E. Thermographic measurements of the spin Peltier effect in metal/yttrium-iron-garnet junction systems. Phys. Rev. B 96, 024424 (2017).

40. Seki, T., Iguchi, R., Takanashi, K. \& Uchida, K. Visualization of anomalous Ettingshausen effect in a ferromagnetic film: direct evidence of different symmetry from spin Peltier effect. Appl. Phys. Lett. 112, 152403 (2018).

41. Uchida, K. et al. Combinatorial investigation of spin-orbit materials using spin Peltier effect. Sci. Rep. 8, 16067 (2018).

42. Barrett, C. S. \& Massalski, T. B. Structure of Metals: Crystallographic Methods, Principles and Data (Pergamon, 1980).

43. Yamazaki, T., Iguchi, R., Ohkubo, T., Nagano, H. \& Uchida, K. Transient response of the spin Peltier effect revealed by lock-in thermoreflectance measurements. Phys. Rev. B 101, 020415(R) (2020).

44. Pi, U. H. et al. Tilting of the spin orientation induced by Rashba effect in ferromagnetic metal layer. Appl. Phys. Lett. 97, 162507 (2010).

45. Kim, J. et al. Layer thickness dependence of the current-induced effective field vector in $\mathrm{Ta}|\mathrm{CoFeB}| \mathrm{MgO}$. Nat. Mater. 12, 240-245 (2013).

46. Garello, K. et al. Symmetry and magnitude of spin-orbit torques in ferromagnetic heterostructures. Nat. Nanotechnol. 8, 587 (2013).

47. Avci, C. O. et al. Interplay of spin-orbit torque and thermoelectric effects in ferromagnet/normal-metal bilayers. Phys. Rev. B 90, 224427 (2014).

48. Lau, Y.-C. \& Hayashi, M. Spin torque efficiency of Ta, W, and Pt in metallic bilayers evaluated by harmonic Hall and spin Hall magnetoresistance measurements. Jpn. J. Appl. Phys. 56, 0802B5 (2017).
49. Nguyen, M.-H., Ralph, D. C. \& Buhrman, R. A. Spin torque study of the spin Hall conductivity and spin diffusion length in platinum thin films with varying resistivity. Phys. Rev. Lett. 116, 126601 (2016).

50. Uchida, K., Daimon, S., Iguchi, R. \& Saitoh, E. Observation of anisotropic magneto-Peltier effect in nickel. Nature 558, 95-99 (2018).

\section{Acknowledgements}

The authors thank I. Narita for technical support to do the composition analysis and M. Isomura for substrate preparation. This work was supported by JSPS KAKENHI Grantin-Aid for Scientific Research (S) (JP18H05246), Grant-in-Aid for Scientific Research (B) (JP19H02585), Grant-in-Aid for Scientific Research (A) (JP20H00299), and JST CREST "Creation of Innovative Core Technologies for Nano-enabled Thermal Management" (JPMJCR17I1). The device fabrication was partly carried out at the Cooperative Research and Development Center for Advanced Materials, IMR, Tohoku University.

\section{Author contributions}

T.S. and K.U. conceived the idea, and planned and supervised this study. H.M. and R.M. carried out the sample preparation and the structural characterization with the help from Y.S., K.U., and T.S. The LIT measurement and its analysis were performed by H.M. and K.U. with input from R.I., and the harmonic Hall measurement was done by H.M. and Y.C.L. T.S. wrote the paper with input from K.U., R.M., Y.C.L., R.I. and K.T. All of the authors contributed to the understanding of physical mechanism.

\section{Competing interests}

The authors declare no competing interests.

\section{Additional information}

Supplementary information is available for this paper at https://doi.org/10.1038/s43246020-00076-0.

Correspondence and requests for materials should be addressed to T.S. or K.U.

Reprints and permission information is available at http://www.nature.com/reprints

Publisher's note Springer Nature remains neutral with regard to jurisdictional claims in published maps and institutional affiliations.

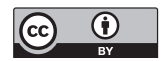

Open Access This article is licensed under a Creative Commons Attribution 4.0 International License, which permits use, sharing, adaptation, distribution and reproduction in any medium or format, as long as you give appropriate credit to the original author(s) and the source, provide a link to the Creative Commons license, and indicate if changes were made. The images or other third party material in this article are included in the article's Creative Commons license, unless indicated otherwise in a credit line to the material. If material is not included in the article's Creative Commons license and your intended use is not permitted by statutory regulation or exceeds the permitted use, you will need to obtain permission directly from the copyright holder. To view a copy of this license, visit http://creativecommons.org/ licenses/by/4.0/.

(C) The Author(s) 2020 